\title{
On symplectic and multisymplectic schemes for the KdV equation
}

\author{
U. M. Ascher* R. I. McLachlan ${ }^{\dagger}$
}

May 7, 2004

\begin{abstract}
We examine some symplectic and multisymplectic methods for the notorious Korteweg-de Vries equation, with the question whether the added structure preservation that these methods offer is key in providing high quality schemes for the long time integration of nonlinear, conservative partial differential equations. Concentrating on 2nd order discretizations, several interesting schemes are constructed and studied.

Our essential conclusions are that it is possible to design very stable, conservative difference schemes for the nonlinear, conservative $\mathrm{KdV}$ equation. Among the best of such schemes are methods which are symplectic or multisymplectic. Semi-explicit, symplectic schemes can be very effective in many situations. Compact box schemes are effective in ensuring that no artificial wiggles appear in the approximate solution. A family of box schemes is constructed, of which the multisymplectic box scheme is a prominent member, which are particularly stable on coarse space-time grids.
\end{abstract}

Keywords: Symplectic method, Multisymplectic method, Korteweg-de Vries equation, Box scheme, Hamiltonian system

\section{Introduction}

The design and development of symplectic methods for Hamiltonian ordinary differential equations (ODEs) has yielded very powerful numerical

\footnotetext{
* Department of Computer Science, University of British Columbia, Vancouver, BC, V6T 1Z4, Canada. (ascher@cs.ubc.ca). Supported in part under NSERC Research Grant 84306.

${ }^{\dagger}$ IFS, Massey University, 11-222, Palmerston North, New Zealand. (R.McLachlan@massey.ac.nz)
} 
schemes with beautiful geometric properties. Symplectic and other symmetric methods have been noted for their superior performance, especially for long time integration. See, e.g., the books and monographs [20, 4, 9] and the many references therein. Recall that Hamiltonian systems describe, for instance, the motion of frictionless, energy conserving mechanical systems. Thus, they possess marginal stability, which corresponding symplectic numerical schemes mimic. This "living at the edge of stability" is enabled, at least for sufficiently small (and possibly many!) time steps, by the implied geometric structure that such discrete schemes conserve.

On the other hand, it has long been known that conservative discretization schemes for nonlinear, nondissipative partial differential equations (PDEs) governing wave phenomena tend to become numerically unstable, and dissipation has subsequently been routinely introduced into such numerical schemes. See, e.g., the books $[19,8]$, which describe the seminal work of Kreiss [12] and much more. For nonlinear problems of this type, in particular, conservative difference schemes are known to occasionally yield numerical solutions which at first look fine, but at a later time may suddenly explode - see Example 2.1 below. Consequently, non-dissipative schemes were discouraged, especially for long time integration. Typical work on pseudospectra, e.g. [21], when applied to stability studies of ODEs, also must assume that eigenvalues are placed off the imaginary axis and into the left half plane, so that sufficiently small circles of stability can be drawn around them: In the context of Hamiltonian systems this corresponds to using a slightly dissipative discretization scheme.

Thus, the common beliefs of two established communities seem headed to a clash upon considering symplectic time discretizations for Hamiltonian semi-discretizations and multisymplectic discretizations of certain Hamiltonian PDEs. The purpose of this paper is to examine this situation numerically, attempting to see whether carefully designed, conservative finite difference and finite volume discretizations can remain stable and deliver sharp solution profiles for a long time, and if yes then to what extent symplecticity structure is essential in such methods.

We therefore consider some symplectic and multisymplectic methods for the notorious Korteweg-de Vries (KdV) equation. Following [1] we study semi-explicit symplectic and fully implicit multisymplectic, 2nd order difference schemes. We demonstrate their accuracy and qualitative properties after many time steps. Some such schemes, especially those based on compact discretizations in both space and time, remain remarkably stable over a wide range of problem and grid parameters (including coarse space-time grids), and we investigate this further using steady state analysis and a dispersion analysis.

This article builds on the work reported in [1], but it concentrates more on the broader question posed above. Section 4.2 develops a different, 
finite volume view of the narrow box scheme (8) which then proves useful in Section 6. The numerical examples are new, as is Section 6.1, and so are the more general box schemes (10) and resulting minor improvements such as (11). Indeed, there is an entire family of box schemes with similar stability and accuracy properties to those of the multisymplectic box scheme (6). We summarize our conclusions in the last section of the article.

\section{The $\mathrm{KdV}$ equation}

The KdV equation is given by

$$
\begin{aligned}
u_{t} & =\alpha\left(u^{2}\right)_{x}+\rho u_{x}+\nu u_{x x x} \\
& =V^{\prime}(u)_{x}+\nu u_{x x x}, \quad V(u)=\frac{\alpha}{3} u^{3}+\frac{\rho}{2} u^{2} .
\end{aligned}
$$

We assume given initial conditions $u(x, 0)=u^{0}(x)$ and periodic boundary conditions. See, e.g., [6] for an analytical treatment.

For this famous equation we consider finite volume and finite difference discretizations on a fixed grid with step sizes $\Delta x, \Delta t$ in space and time, respectively. Before we start this, though, we must explain the choice of the $\mathrm{KdV}$ equation as our testbed.

\subsection{Why KdV?}

There are several good reasons to use the KdV equation as a prototype for our comparative study.

- It is a model nonlinear hyperbolic equation with smooth solutions for all times.

- It is non-dissipative (although, unlike, e.g., the advection equation, it is dispersive). Thus, the $\mathrm{KdV}$ equation is a natural test bed for comparing conservative vs dissipative discretizations.

- The KdV equation (1) is notorious: a lot of previous attention has been devoted to it (indeed, over 1001 papers are cited in MathSciNet). Moreover, it is well-known that unexpected, "nonlinear" instabilities occasionally arise for reasonable-looking finite difference discretizations. Let us consider a corresponding example before continuing with our reasoning.

Example 2.1 (An unexpected instability) The following explicit, leap-frog scheme was proposed in the 1960's [23]. With

$$
\mu=\frac{\Delta t}{\Delta x}
$$


the scheme at $x=x_{i}=i \Delta x, t=t_{n}=n \Delta t$ reads

$$
\begin{aligned}
u_{i}^{n+1} & =u_{i}^{n-1}+\frac{2 \alpha \mu}{3}\left(u_{i-1}^{n}+u_{i}^{n}+u_{i+1}^{n}\right)\left(u_{i+1}^{n}-u_{i-1}^{n}\right) \\
& +\rho \mu\left(u_{i+1}^{n}-u_{i-1}^{n}\right)+\frac{\nu \mu}{(\Delta x)^{2}}\left(u_{i+2}^{n}-2 u_{i+1}^{n}+2 u_{i-1}^{n}-u_{i-2}^{n}\right) .
\end{aligned}
$$

Let us introduce the stencil notation used throughout this paper, which for the above scheme reads

$$
\left[\begin{array}{c}
1 \\
0 \\
-1
\end{array}\right] u=\mu\left(\frac{2 \alpha}{3}\left[\begin{array}{lll}
1 & 1 & 1
\end{array}\right] u+\rho\right)\left[\begin{array}{lll}
-1 & 0 & 1
\end{array}\right] u+\frac{\nu \mu}{(\Delta x)^{2}}\left[\begin{array}{lllll}
-1 & 2 & 0 & -2 & 1
\end{array}\right] u .
$$

Freezing coefficients and applying von Neumann's constant coefficient stability analysis, we obtain that the time step must be restricted to satisfy

$$
\Delta t<\Delta x /\left[\frac{|\nu|}{(\Delta x)^{2}}+2\left|\alpha u_{\max }\right|+|\rho|\right] .
$$

Zhao \& Qin [24] chose the data

$$
\begin{aligned}
& \nu=-0.022^{2}, \alpha=-0.5, \rho=0 \\
& u^{0}(x)=\cos (\pi x), \quad u(0, t)=u(2, t) .
\end{aligned}
$$

The solution profile at different times is displayed in Figure 1. The authors [24] tried various $\Delta x, \Delta t$ combinations satisfying the linear stability bound. Yet they always obtained solution blowup for $t>21 / \pi$.

Indeed, setting $\Delta x=.01$ and $\Delta t=.0001$ the solution calculated using MATLAB standard arithmetic with this explicit scheme is qualitatively correct for a while. But around $t=5$, after about 50,000 time steps (!) enough error accumulates in the solution so that the linear stability bound becomes violated. After a few more steps the solution blows up.

The KdV equation is well-known to satisfy a lot of conservation laws. We highlight, in particular, three properties; see [1] for more detail:

- Let $\|u(t)\|^{2}=\int u^{2}(x, t) d x$. Then, for $\rho=0$, it is easy to see norm preservation

$$
\|u(t)\|=\left\|u^{0}\right\|, \quad \forall t .
$$


(For $\rho \neq 0$ there is a corresponding conservation property which looks more cumbersome without being meaningfully different. See, e.g., [1].)

- The KdV equation is a Hamiltonian PDE (e.g., [15, 4]), viz., it can be written as

$$
u_{t}=\frac{\partial}{\partial x} \frac{\delta H}{\delta u}, \quad H=\int\left(V(u)-\frac{\nu}{2}\left(u_{x}\right)^{2}\right) d x .
$$

- The KdV equation has a multisymplectic structure, see $[2,3,24,1]$. This arises from writing it as

$$
L \mathbf{z}_{t}+K \mathbf{z}_{x}=\nabla S(\mathbf{z})
$$

where $K$ and $L$ are antisymmetric, constant matrices, and

$$
\begin{aligned}
\mathbf{z}= & (\phi, u, v, w)^{T} \\
& \phi_{x}=u, v=-\nu u_{x}, w=v_{x}+\frac{1}{2} \phi_{t}-V^{\prime}(u) .
\end{aligned}
$$

Rather than getting technical, let us simply say that for us, "Multisymplectic $=$ symplectic in both space and time".

These properties may be used to design different discretizations for (1), and the question then is what stability and qualitative accuracy properties are obtained. Let us now continue enumerating interesting properties of $\mathrm{KdV}$.

- When $|\nu| \ll 1$ the PDE (1) is somehow close to the Burgers equation (the dispersion limit). See, e.g., [10, 13]. But Burgers can have shocks, whereas the solution of (1) remains always smooth. For the Burgers equation a non-dissipative scheme cannot avoid developing wiggles as soon as a shock forms [10, 22]. Thus, interesting phenomena crop up when approximating (1) with small $\nu$ using large $\Delta x$.

- When $|\nu|$ is not small the term $\nu u_{x x x}$ leads to stiffness: Fast waves can destabilize schemes. Often these fast waves are not resolved in time.

- Many recent schemes which have appeared in the literature are fully implicit (see, e.g., [4]). But this can be cumbersome! On the other hand, explicit schemes are too limited. So, we considered in [1] a semi-explicit scheme: see Section 3.2 below.

- Symplectic discretizations may not be compact, whereas multisymplectic discretizations are. There is no such difference, e.g., for the classical wave equation. Here we can therefore ask, is the extra structure preservation worthwhile? 


\subsection{Why not KdV?}

There are also reasons that make the choice of the $\mathrm{KdV}$ equation as a prototype for our study less ideal.

- The large amount of previous attention is not only a good thing, it is also distracting to some. In this work we are pursuing only the problem statement provided in Section 1, and thus many good methods and analyses are necessarily left unmentioned.

- $\mathrm{KdV}$ is completely integrable, which is uncharacteristic of nonlinear hyperbolic PDEs. This lessens its importance as a prototype equation to study.

- It has only one space variable! Our comparative study here and in [1] does take advantage of this special feature.

Despite the negatives there are sufficiently many positives to hopefully make the present study of interest.

\section{Schemes based on a classical semi- discretization}

The usual semi-discretization in space reads

$$
\begin{aligned}
u_{t} & =\frac{\alpha}{2 \Delta x}\left(\theta\left[\begin{array}{lll}
-1 & 0 & 1
\end{array}\right] u^{2}+2(1-\theta) u\left[\begin{array}{lll}
-1 & 0 & 1
\end{array}\right] u\right) \\
& +\frac{\rho}{2 \Delta x}\left[\begin{array}{lllll}
-1 & 0 & 1
\end{array}\right] u+\frac{\nu}{2(\Delta x)^{3}}\left[\begin{array}{lllll}
-1 & 2 & 0 & -2 & 1
\end{array}\right] u
\end{aligned}
$$

where $\theta$ is a parameter.

The choice $\theta=1$ yields a Hamiltonian system arising from approximating

$$
H_{\Delta}=\Delta x \sum_{i}\left(V\left(u_{i}\right)-\frac{\nu}{2(\Delta x)^{2}}\left(u_{i+1}-u_{i}\right)^{2}\right)
$$

whereas the choice $\theta=2 / 3$ yields discrete norm preservation

$$
\|u(t)\|_{\Delta}^{2}=\Delta x \sum_{i} u_{i}^{2}=\|u(0)\|_{\Delta}^{2}
$$

(see, e.g. [22]). Thus, one must make a choice regarding which of these two properties to preserve. However, as it turns out, there does not seem to be a major qualitative difference in practice between choosing one of these alternatives or the other. In particular, replacing $\theta=2 / 3$ by $\theta=1$ in the explicit leap-frog scheme of Example 2.1 does not have a significant impact on that scheme's instability. 


\subsection{Full discretization: midpoint}

We next consider applying time discretization to the large ODE system (4). An explicit scheme is usually too limited, as we have seen, and we abandon any such possibility. On the other hand, the implicit midpoint rule preserves the properties of the semi-discretization: With $\theta=1$, midpoint yields a symplectic scheme (because the ODE (4) is Hamiltonian and midpoint is a symplectic method), whereas with $\theta=2 / 3$, midpoint yields a normpreserving scheme,

$$
\left\|u^{n}\right\|_{\Delta}^{2}=\left\|u^{0}\right\|_{\Delta}^{2} \quad \forall n
$$

(because midpoint reproduces quadratic invariants).

In the sequel, as in [1], we have used mostly the symplectic midpoint scheme, although qualitatively similar results are normally obtained also for the norm preserving version. (For an exception of sorts see Example 5.3 and Figure 4.)

In numerical experiments the implicit midpoint scheme stays stable over a wide range of parameters for a long time. For a given discretization grid it is often one of the more accurate among the schemes considered here and in [1]. However, it does develop tiny spatial wiggles for relatively large $\Delta x, \Delta t$, and it can even become unstable in some extreme cases and blow up. (The $l_{2}$-stability for $\theta=2 / 3$ does not guarantee stability for $u_{x}$, see Example 5.3. And when a sharp-sawtooth approximate solution tries to move fast the nonlinear solver eventually falls apart.) We will take this up in Section 6. Meanwhile, let us concentrate on three other schemes.

\subsection{A semi-explicit, symplectic method}

Fully implicit schemes are cumbersome. One must solve large systems of nonlinear equations at each time step. Even if using only one Newton iteration per time step (which can be done with decent accuracy only when using relatively small time steps, and may lose symplecticity) we must form as well as solve a large linear system every time step or two.

So, in [1] we considered a symplectic, semi-explicit scheme, where the nonlinear part of the $\mathrm{KdV}$ equation is discretized explicitly and the rest is discretized implicitly. See also [14]. Starting from the Hamiltonian semidiscretization $u_{t}=D \nabla H_{\Delta}(u), D=\left[\begin{array}{lll}-1 & 0 & 1\end{array}\right] /(2 \Delta x)$, we split the Hamil- 
tonian into three parts:

$$
\begin{aligned}
H_{\Delta} & =\Delta x \sum_{i}\left(V\left(u_{i}\right)-\frac{\nu}{2(\Delta x)^{2}}\left(u_{i+1}-u_{i}\right)^{2}\right) \\
& =\Delta x\left(\sum_{i} V\left(u_{2 i}\right)+\sum_{i} V\left(u_{2 i+1}\right)-\sum_{i} \frac{\nu}{2(\Delta x)^{2}}\left(u_{i+1}-u_{i}\right)^{2}\right) \\
& =H_{\Delta}^{1}+H_{\Delta}^{2}+H_{\Delta}^{3} .
\end{aligned}
$$

The ODE $u_{t}=D \nabla H_{\Delta}^{3}(u)$ has constant coefficients, so we apply the unconditionally stable implicit midpoint discretization. This requires setting up and decomposing the matrix once. For half a time step, let us denote the inverse of this matrix by $M$. To the rest we apply odd/even splitting; e.g., the ODE $u_{t}=D \nabla H_{\Delta}^{1}(u)$,

$$
\left(u_{2 i}\right)_{t}=\frac{1}{2}\left(V^{\prime}\left(u_{2 i+1}\right)-V^{\prime}\left(u_{2 i-1}\right)\right), \quad\left(u_{2 i+1}\right)_{t}=0
$$

is integrated exactly from $t_{n}$ to $t_{n+1}$ for the even part.

Our second-order, semi-explicit symplectic scheme is now obtained by a Strang-type splitting:

$$
\begin{aligned}
& U=M u^{n} ; \\
& U_{\text {odd }}=U_{\text {odd }}+\frac{\Delta t}{4 \Delta x}\left(V^{\prime}\left(U_{\text {odd }+1}\right)-V^{\prime}\left(U_{\text {odd }-1}\right)\right) ; \\
& U_{\text {even }}=U_{\text {even }}+\frac{\Delta t}{2 \Delta x}\left(V^{\prime}\left(U_{\text {even }+1}\right)-V^{\prime}\left(U_{\text {even }-1}\right)\right) ; \\
& U_{\text {odd }}=U_{\text {odd }}+\frac{\Delta t}{4 \Delta x}\left(V^{\prime}\left(U_{\text {odd }+1}\right)-V^{\prime}\left(U_{\text {odd }-1}\right)\right) ; \\
& u^{n+1}=M U .
\end{aligned}
$$

A constant coefficient (von Neumann) stability analysis demands restricting the time step to

$$
\Delta t<\Delta x /\left[2\left|\alpha u_{\max }\right|+|\rho|\right] .
$$

In another small variation which we use, one can also integrate the $\rho u_{x}$ term implicitly without extra cost, and drop $\rho$ from the stability bound.

The selling feature of this semi-explicit scheme is that the work per time step is roughly an order of magnitude less than for a fully implicit method! Just how much faster the semi-explicit scheme is depends of course on various factors, including the implementation details of the nonlinear solver for a fully implicit scheme. 


\section{Box schemes}

All the schemes that we have seen so far use a non-compact semidiscretization of the form (4), so cannot be multisymplectic.

Hence, we applied in [1], following [24], a compact scheme in both $x$ and $t$ centered at a cell (box), or a finite volume, with the corners

$$
\left(x_{i}, t_{n}\right),\left(x_{i+1}, t_{n}\right),\left(x_{i}, t_{n+1}\right),\left(x_{i+1}, t_{n+1}\right) .
$$

Like the implicit midpoint the schemes obtained here are fully implicit; but they are also compact, hence we hope to avoid those extra wiggles that may appear when using (4).

\subsection{Multisymplectic box}

The box scheme, i.e. compact midpoint in both $x$ and $t$ applied to a first order PDE form such as (3), has been around for many years [16, 11, 5]. Recall that KdV can be written as (3). Zhao \& Qin [24] showed, following $[18,3]$, that for $\mathrm{KdV}$ the result is multisymplectic. Eliminating $\phi, v, w$, a 12-point scheme was obtained in [24],

$$
\begin{aligned}
\frac{1}{16 \Delta t}\left[\begin{array}{cccc}
1 & 3 & 3 & 1 \\
0 & 0 & 0 & 0 \\
-1 & -3 & -3 & -1
\end{array}\right] u & =\frac{1}{4 \Delta x}\left[\begin{array}{ccc}
-1 & 0 & 1 \\
-1 & 0 & 1
\end{array}\right] V^{\prime}\left(\frac{1}{4}\left[\begin{array}{cc}
1 & 1 \\
1 & 1
\end{array}\right] u\right) \\
& +\frac{\nu}{4(\Delta x)^{3}}\left[\begin{array}{cccc}
-1 & 3 & -3 & 1 \\
-2 & 6 & -6 & 2 \\
-1 & 3 & -3 & 1
\end{array}\right] u
\end{aligned}
$$

This scheme turns out to be rather stable and wiggle-free in many (though not all) situations, which is rather remarkable for a conservative, two-step, unintuitive scheme!

In [1] we simplified (6) into the 8-point scheme

$$
\begin{aligned}
\frac{1}{8 \Delta t}\left[\begin{array}{cccc}
1 & 3 & 3 & 1 \\
-1 & -3 & -3 & -1
\end{array}\right] u & =\frac{1}{2 \Delta x}\left[\begin{array}{lll}
-1 & 0 & 1
\end{array}\right] V^{\prime}\left(\frac{1}{4}\left[\begin{array}{ll}
1 & 1 \\
1 & 1
\end{array}\right] u\right) \\
& +\frac{\nu}{2(\Delta x)^{3}}\left[\begin{array}{cccc}
-1 & 3 & -3 & 1 \\
-1 & 3 & -3 & 1
\end{array}\right] u
\end{aligned}
$$

Averaging the 8-point scheme (7) at time levels $n$ and $n-1$ gives the 12point scheme (6). So, using (7) or (6) we obtain identical results up to roundoff error level if applying the 8-point scheme to initialize the 12-point scheme.

We call (7) our multisymplectic box. 


\subsection{Fully implicit narrow box}

Already Reich [17] has observed that the box scheme which preserves multisymplectic properties can be obtained upon applying standard finite volume techniques to a multisymplectic (3). But now, let us apply a standard finite volume discretization directly to the $\mathrm{KdV}$ equation in the form (1), in the hope to obtain a more accurate, compact discretization at the price of potentially abandoning multisymplecticity:

1. Write $\mathrm{KdV}$ in divergence form

$$
\begin{aligned}
\operatorname{div}(u, v) \equiv u_{t}+v_{x} & =0 \\
v & =-V^{\prime}(u)-\nu u_{x x} .
\end{aligned}
$$

2. Integrate this divergence free form using the Gauss Divergence Theorem,

$$
\frac{1}{\Delta t}\left(u_{i+1 / 2}^{n+1}-u_{i+1 / 2}^{n}\right)+\frac{1}{\Delta x}\left(v_{i+1}^{n+1 / 2}-v_{i}^{n+1 / 2}\right)=0 .
$$

This formula is exact, where

$$
u_{i+1 / 2}^{n}=\frac{1}{\Delta x} \int_{x_{i}}^{x_{i+1}} u\left(x, t_{n}\right) d x, \quad v_{i}^{n+1 / 2}=\frac{1}{\Delta t} \int_{t_{n}}^{t_{n+1}} v\left(x_{i}, t\right) d t .
$$

3. Apply the usual compact discretization for the second spatial derivative appearing in the definition of $v$,

$$
-v_{i}^{n+1 / 2}=V^{\prime}\left(u_{i}^{n+1 / 2}\right)+\frac{\nu}{(\Delta x)^{2}}\left[\begin{array}{lll}
1 & -2 & 1
\end{array}\right] u_{i}^{n+1 / 2} .
$$

This is a second order approximation.

4. Discretize the line integrals by the trapezoidal rule. This yields the scheme

$$
\begin{aligned}
\frac{1}{2 \Delta t}\left[\begin{array}{cc}
1 & 1 \\
-1 & -1
\end{array}\right] u & =\frac{1}{\Delta x}\left[\begin{array}{ll}
-1 & 1
\end{array}\right] V^{\prime}\left(\frac{1}{2}\left[\begin{array}{l}
1 \\
1
\end{array}\right] u\right) \\
& +\frac{\nu}{2(\Delta x)^{3}}\left[\begin{array}{cccc}
-1 & 3 & -3 & 1 \\
-1 & 3 & -3 & 1
\end{array}\right] u
\end{aligned}
$$

We call (8) our narrow box scheme. The terms corresponding to first derivatives in (1) have narrower stencils than in (7), whereas the third derivative approximation is the same. The narrow scheme boasts a smaller coefficient in its leading truncation error term than (7) has. 


\section{Some numerical tests}

We have identified four schemes to concentrate on: the implicit midpoint, the semi-explicit symplectic, the multisymplectic box and the narrow box. Next, we perform some numerical tests. For much more, see [1].

Example 5.1 (Example 2.1 again) Consider the same example as the one used to demonstrate instability before.

Noting that $2\left|\alpha u_{\max }\right| \approx 2.6$, we take this to be the Courant number for the semi-explicit method. Thus, we set

$$
\mu=0.2
$$

both here and in Example 5.3 below.

Here we take $\Delta x=.005, \Delta t=.001$, and record relative errors at $t=5$ and at $t=10$ in the discrete Hamiltonian (5) (denoted Error-Ham) and in the $l_{2}$-norm (denoted Error-norm). Note that these are often underestimators for the maximal pointwise error. The results are displayed in Table 1.

The main observation concerning these results is that the multisymplectic box is the least accurate, and by a significant margin. On the other hand, the semi-explicit, symplectic method shines, given its much smaller computational cost per time step. The narrow box scheme is the most accurate on the given grid.

Example 5.2 (A different example) The following is also taken from [24]. We choose

$$
\begin{aligned}
& \nu=-1, \alpha=-3, \rho=0 \\
& u^{0}(x)=6 \operatorname{sech}^{2}(x), \quad u(-20, t)=u(20, t) .
\end{aligned}
$$

After a short while the solution has two solitons, a tall and narrow one (maximum height $=8$ ) and a shorter, wider one. These pulses both move with time to the right, wrapping around as is usual for periodic boundary conditions. But the taller soliton moves faster, hence the two occasionally merge and then split apart again. See Figure 2 for snapshots at times $t=4$ and $t=100$. 
Turning to the numerical discretization, note that $2\left|\alpha u_{\max }\right| \approx 48$. For the semi-explicit scheme we take $\Delta x=.05, \Delta t=.001$.

But the remaining three fully implicit schemes do not share the stability restriction of the semi-explicit scheme. For them we take $\Delta x=$ $.05, \Delta t=.005$. This yields roughly equal computation time per time step for all schemes. Results are recorded in Table 2.

For these parameters the fully implicit midpoint scheme happens to perform poorly. The coarser time step .005 is simply too rough for it, and it displays behaviour that would be typical for a dissipative scheme. (The variant developed in [7] and described in [4] performs similarly.) For the finer time step 001 its accuracy is comparable to that of the others, and the characteristics of the symplectic method re-emerge, but then it can be argued that its computational expense is unjustified.

The other striking effect in the results of Table 2 is that these error underestimators hardly deteriorate for a long time. Note that with $\Delta t=.001$ it takes 100,000 time steps to arrive at $t=100$. These error underestimators thus appear to be too good to be true! Indeed, the pointwise error does deteriorate: The shape of the solitons is preserved by all schemes other than midpoint with $\Delta t=.005$, but their location is not. This well-known numerical dispersion effect is evident in Figure 2. Further experimentation with finer space-time grids indicates that the phase shift is significantly the smallest in the semi-explicit plot Figure 2(a) (where the computation was indeed performed with a smaller time step).

Example 5.3 (Rough grids) We now return to the problem of Example 2.1 and apply our discretization schemes using rough resolutions. By this we mean that the discretization step $\Delta x$ is too large to be able to reconstruct all the solution details visible at $t=10$ in Figure 1. (See also [1] for another detailed example of a similar sort.) We keep $\mu=0.2$ fixed.

At first we set $\Delta x=.02$, thus $\Delta t=.004$. The solutions are graphed in Figure 3.

Note the extra wiggles that appear in the solution curves of those schemes which are based on the semi-discretization (4). The box schemes do not show such wiggles. The narrow box scheme produces a curve which is less smooth but closer to the exact solution, whereas the multisymplectic box scheme produces a very stable, wiggle-free, not very accurate solution. 
Next we make things really rough by setting $\Delta x=1 / 30$, hence $\Delta t=$ 1/150. Now the semi-explicit, symplectic scheme blows up before reaching $t=10$, and so do the narrow box and the symplectic midpoint schemes. The multisymplectic box scheme is the only one which continues to produce smooth, wavy solution profiles which, while having little to do with the exact solution, remain very stable and could look like the exact solution of another problem of the same type; see Figure $4(a)$.

As mentioned earlier, setting $\theta=2 / 3$ rather than $\theta=1$ in (4) and applying implicit midpoint in time produces a scheme which is "unconditionally stable" in the $l_{2}$-sense. For the present example the solution indeed does not blow up in this case, unlike its symplectic comrade. However, it does not look like much more than noise either, even though the $l_{2}$-norm is in relative error of only $3.9 e-8$ (which comes from the accuracy tolerance of the nonlinear solver); see Figure 4(b).

\section{$6 \quad$ Stability of some box schemes}

The apparent stability of the multisymplectic box scheme, as demonstrated in Example 5.3 for large step sizes, is noteworthy. This is so because the scheme is conservative, and as such only marginally stable. Moreover, symplectic methods are generally expected to do well over a long time using small discretization steps, not large ones; see, e.g., [9]. Is there something magical about the multisymplectic structure?

To analyze this we consider, following [1], the linearized KdV

$$
u_{t}=\hat{\rho} u_{x}+\nu u_{x x x} .
$$

The discretized (9) depends on two parameters,

$$
r=\hat{\rho} \frac{\Delta t}{\Delta x}, \quad s=\frac{\hat{\rho}}{\nu}(\Delta x)^{2} .
$$

The first parameter is the signed Courant number for the semi-explicit method, whereas the second indicates the strength of the first spatial derivative term as compared to the third derivative term over the current spatial grid. 
Extending [1], let us consider the general one-step box discretization

$$
\begin{aligned}
\frac{1}{\Delta t}\left[\begin{array}{cccc}
d & c & c & d \\
-d & -c & -c & -d
\end{array}\right] u & =\frac{\hat{\rho}}{2 \Delta x}\left[\begin{array}{cccc}
-b & -a & a & b \\
-b & -a & a & b
\end{array}\right] u \\
& +\frac{\nu}{2(\Delta x)^{3}}\left[\begin{array}{cccc}
-1 & 3 & -3 & 1 \\
-1 & 3 & -3 & 1
\end{array}\right] u
\end{aligned}
$$

Second order accuracy requires that $a+3 b=1, c+d=1 / 2$, so we really have only two free parameters, $a$ and $c$.

Clearly, the choice $a=1 / 4, c=3 / 8$ yields our multisymplectic box, and the choice $a=1, c=1 / 2$ yields the narrow box. But other possibilities arise. For instance, recalling the finite volume derivation of the narrow box scheme we realize that a wider, 4-point spatial stencil, required anyway by the third derivative discretization, can be used to design a more accurate quadrature of $u_{i+1 / 2}$, replacing the trapezoidal rule in this task. This yields the value $c=\frac{13}{24}$.

\subsection{The steady state case}

The situation for the general box scheme (10) becomes very simple if $u_{t}=0$. Although this is not expected to happen for interesting $\mathrm{KdV}$ equations, the analysis does provide some indications as to what may be expected, at least when $\Delta t$ is relatively large.

For (10) we obtain a linear difference scheme with the characteristic polynomial

$$
p(\zeta)=(\zeta-1)\left[(s b+1)\left(\zeta^{2}+1\right)+(s(a+b)-2) \zeta\right] .
$$

The scheme is stable only if all roots of the characteristic polynomial are in the unit circle, which occurs if

$$
\begin{cases}s<\frac{4}{1-4 b}, & b<1 / 4, \\ \text { Unconditionally, } & b \geq 1 / 4 .\end{cases}
$$

Note that $b \geq 1 / 4$ implies $a \leq 1 / 4$. Moreover, the larger $b$ the wider the stencil of the first derivative discretization, so the less accurate is the corresponding truncation error term.

For the narrow box scheme we have $b=0$, hence the stability condition is $s<4$, which is normally very generous. The same condition can be easily shown to hold also for the semi-explicit discretization (4). We have therefore obtained the following results:

- The most accurate, unconditionally stable box semi-discretization at steady state is the multisymplectic box scheme. 
- For the narrow box, as well as the fully implicit midpoint and the semi-explicit symplectic schemes, stability at steady state holds if

$$
s<4
$$

For Example 2.1, hence also Examples 5.1 and 5.3, representative values are

$\nu=-.022^{2}, \hat{\rho}=-2.6$. Thus,

$$
\begin{array}{rll}
\Delta x=.005 & \Rightarrow & s=0.134 \\
\Delta x=.02 & \Rightarrow & s=2.15 \\
\Delta x=1 / 30 & \Rightarrow & s=5.96 .
\end{array}
$$

In particular, for $\Delta x=1 / 30, s>4$.

\subsection{Dispersion analysis}

The steady state analysis is insufficient, of course, to describe the general situation. Indeed, those extra wiggles that the non-compact schemes display in Figure 3 have not been accounted for. But in [1] we show that a dispersion analysis does account for the more general situation.

Thus, we substitute a solution of the form

$$
u_{j}^{n}=e^{\imath(\xi j+\omega n)}=e^{\imath[(\xi / \Delta x)(j \Delta x)+(\omega / \Delta t)(n \Delta t)]}=u(j \Delta x, n \Delta t),
$$

and plot dispersion curves of frequency $\omega$ vs wave number $\xi$ for the linearized KdV (9), the semi-discretization (4), the fully implicit midpoint, and the two box schemes.

Dispersion curves at $s=.134, \mu=0.2$ are displayed in Figure 5(a). We see that the box schemes exhibit excellent behaviour, qualitatively preserving the exact dispersion relation. For the multisymplectic box this is a corollary of a general proposition for (3) proved in [1]. On the other hand, the schemes based on (4) do support combinations of small frequencies and large wave numbers which are not supported by the exact linear KdV.

This defect of the non-compact schemes becomes more serious for larger $s$. In Figure 5(b) we display dispersion curves at $s=2.15, \mu=0.2$. Now, whereas the box schemes are still qualitatively correct, for the non-compact schemes there is a whole range of high wavenumbers which is supported by low frequencies. Since the approximated solution is rich in low frequencies we may expect artificial wiggles due to corresponding high wave numbers in the approximate solutions obtained by schemes which are based on (4). This is indeed borne out by the results reported in Example 5.3, see especially Figure 3. 
Having extended [1] by considering also other box schemes of the form (10) we next check what happens if we let $a$ and $c$ take on different values, for instance $a=1 / 8$ and/or $c=13 / 24$. It turns out that the obtained schemes produce qualitatively similar dispersion curves.

Finally, we consider a case where $s>4$. Dispersion curves at $s=$ $5.96, \mu=0.2$ are displayed in Figure 5(c). Now only the multisymplectic box scheme sticks with the correct dispersion curve, qualitatively speaking. These observations again correspond very well to our findings in Example 5.3, particularly Figure 4.

It is tempting to conclude at this point that there is indeed something special, not quite explained by theory, by which multisymplecticity yields supreme stability! However, it turns out that other box schemes with $a \leq$ $1 / 4$ (e.g. $a=1 / 8$ ), behave qualitatively the same as the multisymplectic box with $a=1 / 4$ does. Moreover, other box schemes of the form (10) with $c=13 / 24$ produce qualitatively similar dispersion curves, depending only on the value of $a$. Indeed, for $u_{t}$ we have a variant of the midpoint rule, regardless of the value of $c$, so varying $c$ is not expected to affect stability significantly.

Example 6.1 Running the experiments of Examples 5.2, 5.1 and 5.3 using schemes with $a \leq 1 / 4$ and $c=13 / 24$ yields qualitatively similar results to those previously discussed.

In Table 3 we display results using $c=13 / 24$, comparing them to previous ones from Tables 1 and 2. Clearly, choosing $c=13 / 24$ appears to improve accuracy in a minor way, especially in terms of conserving the $l_{2}$-norm, without compromising stability.

\section{Conclusions}

- Symplectic and multisymplectic methods can provide successful conservative schemes for the KdV equation! Specifically, they can stay remarkably stable and preserve sharp solution features over a very long time.

- A symplectic, semi-explicit scheme is proposed which combines implicit midpoint for the $u_{x x x}$ term with an explicit odd/even splitting for the Burgers term. This usually performs well when the time step 
is restricted by a constant coefficient analysis for the explicit part alone.

- A multisymplectic box scheme has been simplified and studied compared to a narrow box scheme. These are fully implicit, hence more expensive than the semi-explicit scheme, but they allow larger time steps and remain remarkably stable for a wide variety of parameters.

- A steady state stability analysis for the linearized KdV indicates unconditional stability for a family of box schemes of which the multisymplectic box is the most accurate. For other schemes including the narrow box there is conditional stability with a generous condition.

- A dispersion analysis suggests advantage to the box schemes over schemes based on non-compact spatial discretization. The latter may produce artificial wiggles into the solution. Even for conditionally stable box schemes the dispersion curves indicate trouble when the steady state stability condition is violated.

- The multisymplectic box scheme is not very accurate. It appears inferior to the narrow box scheme in this regard. However, the accuracy of both schemes can be somewhat improved without affecting their stability properties. In particular, the scheme

$$
\begin{aligned}
\frac{1}{24 \Delta t}\left[\begin{array}{cccc}
-1 & 13 & 13 & -1 \\
1 & -13 & -13 & 1
\end{array}\right] u= & \frac{1}{2 \Delta x}\left[\begin{array}{lll}
-1 & 0 & 1
\end{array}\right] V^{\prime}\left(\frac{1}{4}\left[\begin{array}{cc}
1 & 1 \\
1 & 1
\end{array}\right] u\right)+ \\
& \frac{\nu}{2(\Delta x)^{3}}\left[\begin{array}{cccc}
-1 & 3 & -3 & 1 \\
-1 & 3 & -3 & 1
\end{array}\right] u
\end{aligned}
$$

offers an interesting variant of the multisymplectic box.

- It remains unclear whether the concept of multisymplecticity as such is crucial to (approximately) preserve in practical discretizations. The simpler concept of compact discretization in both time and space is probably more important practically. And yet, the multisymplectic method (6) does possess impressive properties. Its nonintuitive discretization of the nonlinear $\mathrm{KdV}$ term is best in terms of combining accuracy with unconditional stability, and the scheme (11) may be regarded as a "relative" of the multisymplectic scheme.

\section{Acknowledgments:}

The first author thanks Andre Nachbin for many helpful discussions. He also thanks IMPA for its hospitality and support. 


\section{References}

[1] U. M. Ascher and R. I. McLachlan. Multisymplectic box schemes and the Korteweg-de Vries equation. Numerical Algorithms, 2003. to appear.

[2] T. J. Bridges. Multi-symplectic structures and wave propagation. Math. Proc. Camb. Phil. Soc., 121:147-190, 1997.

[3] T. J. Bridges and S. Reich. Multi-symplectic integrators: numerical schemes for Hamiltonian PDEs that conserve symplecticity. Phys. Lett. A, 284 (4-5):184-193, 2001.

[4] C.J. Budd and M.D. Piggott. Geometric integration and its applications. Handbook of Numerical Analysis vol. XI, pages 35-139, 2003. P. G. Ciarlet and F. Cucker (eds.), North-Holland, Amsterdam.

[5] J.-P. Croisille. Keller's box-scheme for the one-dimensional stationary convection-diffusion equation. Computing, 68(1):37-63, 2002.

[6] P. G. Drazin and R. S. Johnson. Solitons: An Introduction. Cambridge University Press, 1989.

[7] D. Furihata. Finite difference schemes for $\partial u / \partial t=(\partial / \partial x)^{\alpha} \delta g / \delta u$ that inherit energy conservation or dissipation property. J. Comp. Phys., 156:181-205, 1999.

[8] B. Gustafsson, H.-O. Kreiss, and J. Oliger. Time Dependent Problems and Difference Methods. Wiley \& Sons, New York, 1995.

[9] E. Hairer, C. Lubich, and G. Wanner. Geometric Numerical Integration. Springer, 2002.

[10] T.Y. Hou and P.D. Lax. Dispersive approximation in fluid dynamics. Comm. Pure Appl. Math., 44:1-40, 1991.

[11] H. B. Keller. A new difference scheme for parabolic problems. In Numerical Solution of Partial Differential Equations, II (SYNSPADE 1970), pages 327-350. Academic Press, New York, 1971.

[12] H.-O. Kreiss. On difference approximations of the dissipative type for hyperbolic differential equations. Comm. Pure Applied Math., 17:335353, 1964.

[13] P.D. Lax and C.D. Levermore. The small dispersion limit of the Korteweg-de Vries equation. Comm. Pure Appl. Math., 36:253-290, 571-593, 809-829, 1983. 
[14] R. I. McLachlan and G. R. W. Quispel. Splitting methods. Acta Numerica, 11:341-434, 2002.

[15] R.I. McLachlan. Symplectic integration of hamiltonian wave equations. Numer. Math., 66:465-492, 1994.

[16] A. Preissmann. Propagation des intumescences dans les canaux et rivières. In First Congress French Assoc. for Computation, 1961. Grenoble.

[17] S. Reich. Finite volume methods for multi-symplectic PDEs. BIT, 40:559-582, 2000.

[18] S. Reich. Multi-symplectic Runge-Kutta collocation methods for Hamiltonian wave equations. J. Comput. Phys., 157(2):473-499, 2000.

[19] R.D. Richtmyer and K.W. Morton. Difference Methods for InitialValue Problems. Wiley, 1967.

[20] J.M. Sanz-Serna and M.P. Calvo. Numerical Hamiltonian Problems. Chapman and Hall, 1994.

[21] L.N. Trefethen. Pseudospectra of linear operators. SIAM Review, 39:383-406, 1997.

[22] C.V. Turner and R.R. Rosales. The small dispersion limit for a nonlinear semidiscrete system of equation. Studies Appl. Math., 99:205-254, 1997.

[23] N. J. Zabusky and M. D. Kruskal. Interaction of 'solitons' in a collisionless plasma and the recurrence of initial states. Phys. Rev., 15:240-243, 1965 .

[24] P. F. Zhao and M. Z. Qin. Multisymplectic geometry and multisymplectic Preissmann scheme for the KdV equation. J. Phys. A, 33(18):3613-3626, 2000. 


\begin{tabular}{|cccc|}
\hline Method & $T$ & Error-Ham & Error-norm \\
\hline Multisymplectic Box & 5 & $5.0 e-1$ & $2.1 e-3$ \\
Narrow Box & 5 & $6.0 e-5$ & $4.9 e-4$ \\
Semi-explicit Symplectic & 5 & $3.6 e-3$ & $8.9 e-4$ \\
Implicit midpoint & 5 & $8.4 e-5$ & $8.9 e-4$ \\
\hline Multisymplectic Box & 10 & $4.8 e-1$ & $2.0 e-3$ \\
Narrow Box & 10 & $8.6 e-5$ & $4.8 e-4$ \\
Semi-explicit Symplectic & 10 & $3.7 e-3$ & $8.7 e-4$ \\
Implicit midpoint & 10 & $1.2 e-4$ & $8.7 e-4$ \\
\hline \hline
\end{tabular}

Table 1: Error indicators in Hamiltonian and norm for Example 2.1 using $\Delta x=.005, \Delta t=.001$. 


\begin{tabular}{|ccccc|}
\hline Method & $T$ & $\Delta t$ & Error-Ham & Error-norm \\
\hline Multisymplectic Box & 4 & .005 & $5.8 e-3$ & $8.8 e-4$ \\
Narrow Box & 4 & .005 & $1.5 e-3$ & $2.3 e-4$ \\
Semi-explicit Symplectic & 4 & .001 & $3.5 e-4$ & $4.5 e-4$ \\
Implicit midpoint & 4 & .005 & $6.6 e-2$ & $2.0 e-2$ \\
Implicit midpoint & 4 & .001 & $1.2 e-4$ & $5.0 e-4$ \\
\hline Multisymplectic Box & 100 & .005 & $5.8 e-3$ & $8.9 e-4$ \\
Narrow Box & 100 & .005 & $1.5 e-3$ & $2.3 e-4$ \\
Semi-explicit Symplectic & 100 & .001 & $3.5 e-4$ & $4.5 e-4$ \\
Implicit midpoint & 100 & .005 & $3.7 e-1$ & $1.2 e-1$ \\
Implicit midpoint & 100 & .001 & $1.1 e-4$ & $5.0 e-4$ \\
\hline \hline
\end{tabular}

Table 2: Error indicators in Hamiltonian and norm for Example 5.2 using $\Delta x=.05$. 


\begin{tabular}{|ccccc|}
\hline Example 2.1 & & $T=10$ & $\Delta x=.005$ & $\Delta t=.001$ \\
\hline Method & $a$ & $c$ & Error-Ham & Error-norm \\
\hline Multisymplectic Box & $1 / 4$ & $3 / 8$ & $4.8 e-1$ & $2.0 e-3$ \\
& $1 / 4$ & $13 / 24$ & $4.7 e-1$ & $3.5 e-6$ \\
Narrow Box & 1 & $1 / 2$ & $8.6 e-5$ & $4.8 e-4$ \\
& 1 & $13 / 24$ & $7.7 e-5$ & $3.6 e-6$ \\
Midpoint & & & $1.2 e-4$ & $8.7 e-4$ \\
\hline \hline Example 5.2 & & $T=4$ & $\Delta x=.05$ & $\Delta t=.005$ \\
\hline Method & $a$ & $c$ & Error-Ham & Error-norm \\
\hline Multisymplectic Box & $1 / 4$ & $3 / 8$ & $5.8 e-3$ & $8.8 e-4$ \\
& $1 / 4$ & $13 / 24$ & $5.6 e-3$ & $1.3 e-6$ \\
Narrow Box & 1 & $1 / 2$ & $1.5 e-3$ & $2.3 e-4$ \\
& 1 & $13 / 24$ & $1.4 e-3$ & $3.2 e-7$ \\
\hline \hline
\end{tabular}

Table 3: The effect of choosing $c=13 / 24$. The $l_{2}$-norm is better preserved without any apparent penalty. 
On symplectic and multisymplectic schemes for $K d V$

\section{Figure captions}

Figure 1. Snapshots of the solution for Example 2.1.

Figure 2. Solutions for Example 5.2 at times $t=4$ and $t=100$. Note how crisply all of the conservative schemes except midpoint capture the solitons after a long time integration.

Figure 3. Solutions for Example 2.1 for a rough resolution $\Delta x=.02$ at time $t=10$. Note the wiggles in the non-compact schemes (a) and (b).

Figure 4. Solutions for example 2.1 for a very rough resolution $\Delta x=1 / 30$ at time $t=10$.

Figure 5. Dispersion curves at various values of $s$, with $\mu=0.2$, for the linearized $\mathrm{KdV}$. 


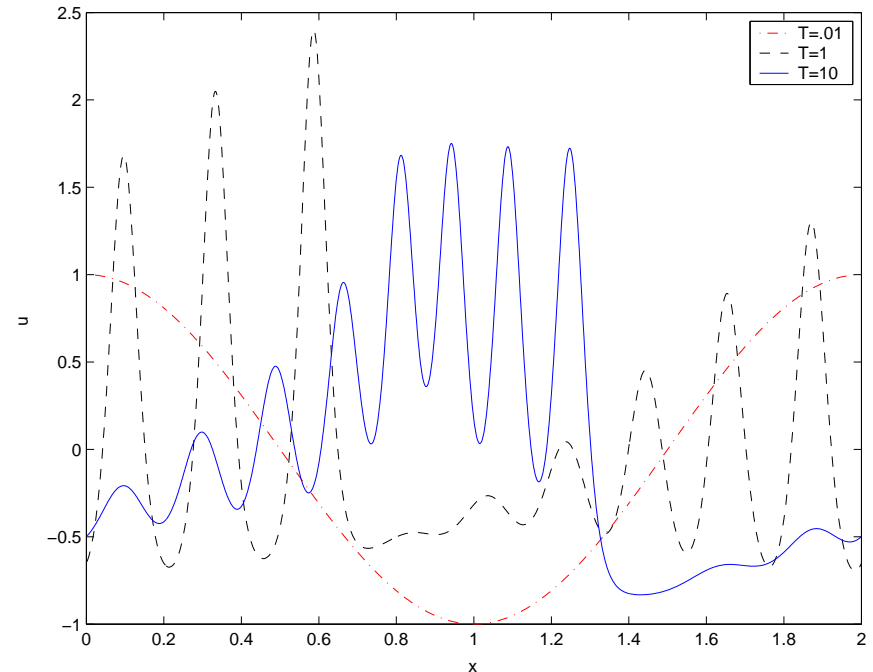

Figure 1: 


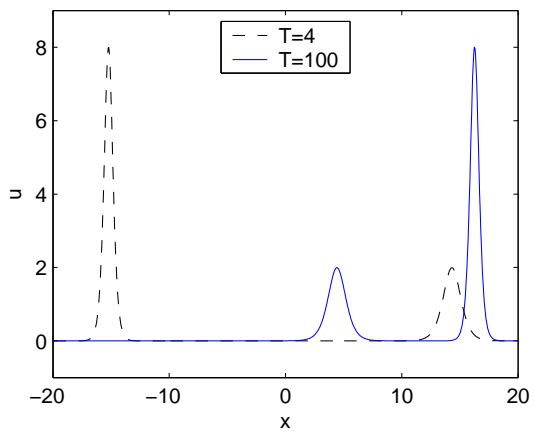

(a) Semi-explicit, symplectic, $\Delta t=$ .001 .

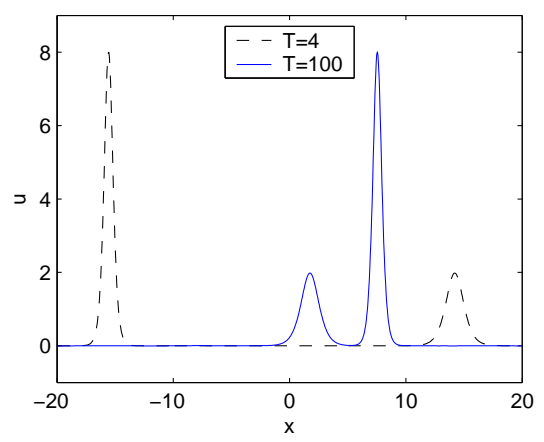

(c) Multisymplectic box, $\Delta t=.005$

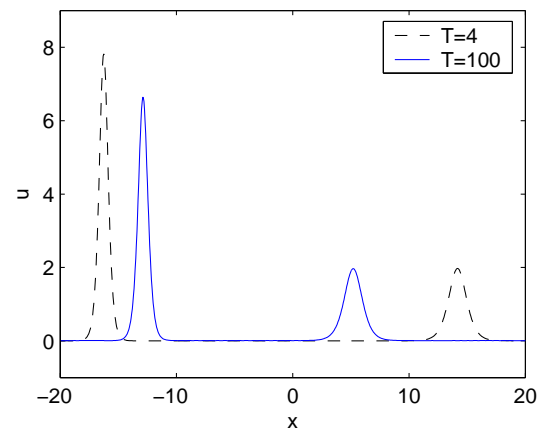

(b) Implicit midpoint, $\Delta t=.005$

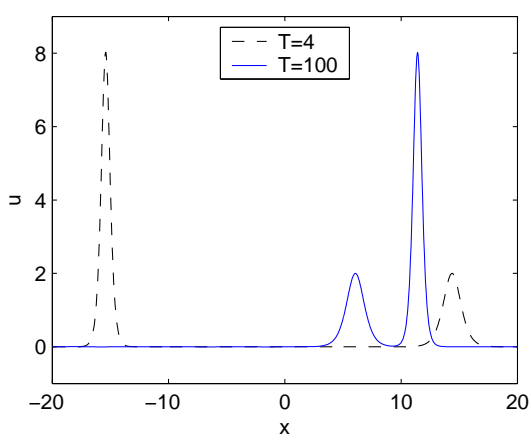

(d) Narrow box, $\Delta t=.005$

Figure 2: 


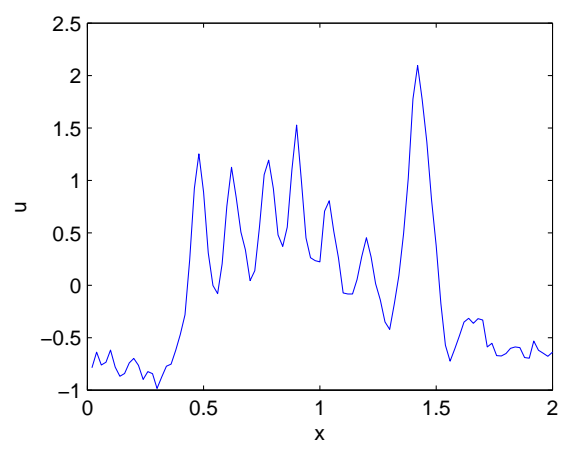

(a) Semi-explicit, symplectic

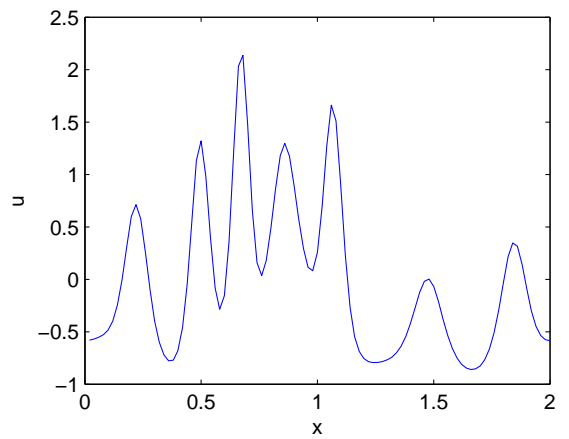

(c) Multisymplectic box

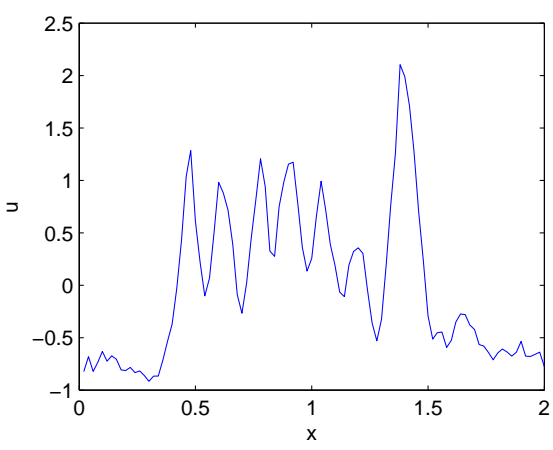

(b) Implicit midpoint

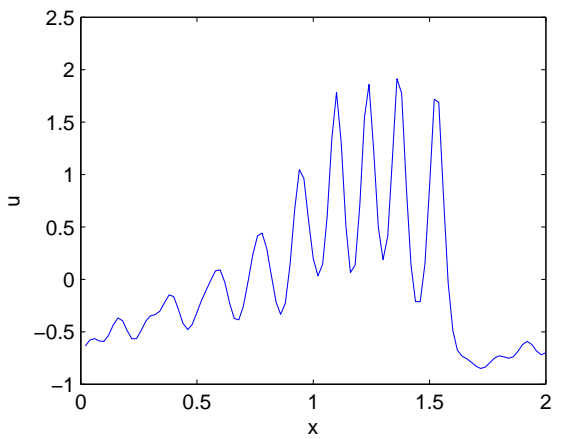

(d) Narrow box

Figure 3: 


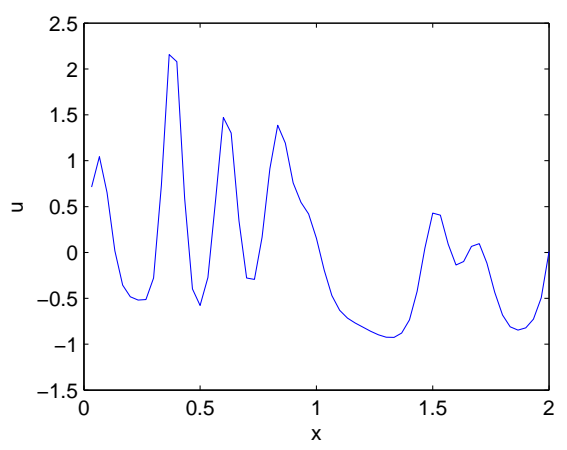

(a) Multisymplectic box

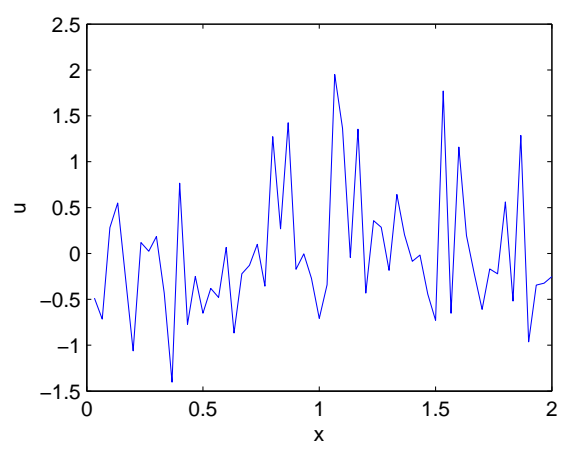

(b) Implicit midpoint $\theta=2 / 3$

Figure 4: 


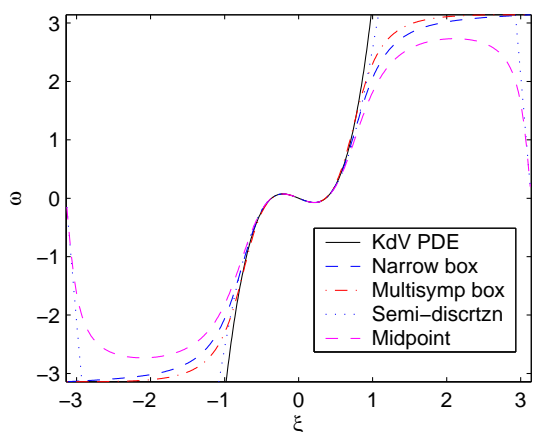

(a) $s=.134$

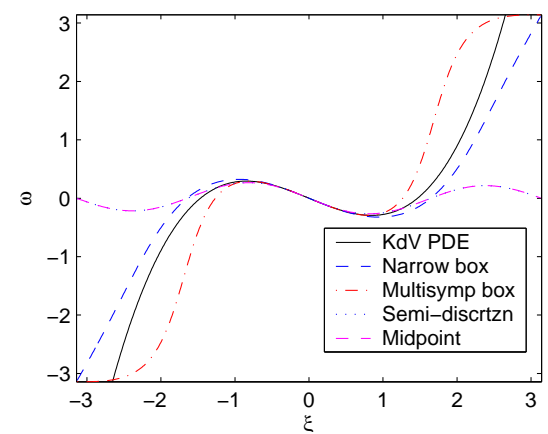

(b) $s=2.15$

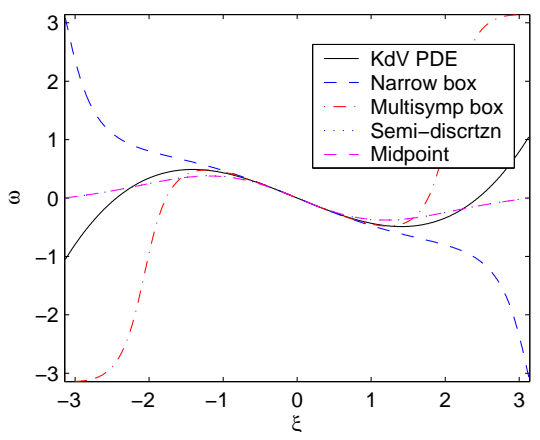

(c) $s=5.96$

Figure 5: 